\title{
MARCINKIEWICZ FUNCTIONS WITH HARDY SPACE KERNELS
}

\author{
AHMAD AL-SALMAN
}

Abstract. In this paper we prove $L^{p}$ estimates of Marcinkiewicz integral operators with kernels in the Hardy space and supported on general subvarieties. The considered subvarieties are of the type that caries partially the polynomial behavior as well as the behavior of convex functions. Results obtained in this paper improve as well as generalize known results.

Mathematics subject classification (2010): 42B20, 42B15, 42B25.

Keywords and phrases: Marcinkiewicz integrals, rough kernels, Hardy space, convex functions, Fourier transform, area integral, Littlewood-Paley $g_{\lambda}^{*}$ functions.

\section{REFERENCES}

[1] A. Al-Salman, Marcinkiewicz functions along flat surfaces with hardy space kernels, J. Integral Equations Appl. 17, 4 (2005).

[2] A. Al-SAlman, $L^{p}$ estimates of singular integral operators of convolution type with rough kernels, Ph. D Thesis, University of Pittsburgh, 1999.

[3] A. Al-Salman, Y. PAN, Singular integrals with rough kernels in $\operatorname{Llog}^{+} L\left(\mathbb{S}^{n-1}\right)$, J. London. Math. Soc. (2) 66 (2002) 153-174.

[4] A. Al-Salman, H. Al-QAssem, Singular integrals along flat curves with kernels in the Hardy Space $H^{1}\left(\mathbb{S}^{n-1}\right)$, Proc. of the conference in honour of professor Georgii Litvinchuk "Factorization, Singular Operators and Related Problems", Madeira, Portugal (2002), 1-12.

[5] A. Al-Salman, H. Al-QAssem, Integral operators of Marcinkiewicz type, J. Integral Equations Appl. 14, 4 (2002).

[6] A. Benedek, A. Calderón, R. Panzone, Convolution operators on Banach space valued functions, Proc. Nat. Acad. Sci. USA 48 (1962) 356-365.

[7] J. Chen, D. FAn, Y. PAN, A note on a Marcinkiewicz integral operator, Math. Nachr. 227 (2001), $33-42$.

[8] R. R. Colfman, G. Weiss, Extensions of Hardy spaces and their use in Analysis, Bull. Amer. Math. Soc. 83 (1977), 569-645.

[9] L. Colzani, Hardy spaces on Sphere, Ph. D. Thesis, Washington University, St. Louis, Mo, 1982.

[10] Y. Ding, D. FAN, Y. PAN, $L^{p}$ boundedness of Marcinkiewicz integrals with Hardy space function kernel, Acta. Math. Sinica (English Series) 16 (2000), 593-600.

[11] J. Duonndikoetxea, J. L. Rubio De Francia, Maximal and singular integral operators via Fourier transform estimates, Invent. Math. 84 (1986), 541-561.

[12] D. FAN, Y. PAN, Singular integrals with rough kernels supported by subvarieties, Amer. J. Math. 119 (1997), 799-839.

[13] Hörmander, Translation invariant operators, Acta Math. 104 (1960), 93-139.

[14] W. Kim, S. Wainger, J. Wright, And S. Ziesler, Singular integrals and Maximal functions associated to surfaces of revolution, Bull. London Math. Soc. 28 (1996), 291-296.

[15] E. M. Stein, On the function of Littlewood-Paley, Lusin and Marcinkiewicz, Trans. Amer. Math. Soc. 88 (1958), 430-466.

[16] E. M. SteIn, Harmonic Analysis: Real-Variable Methods, Orthogonality and Oscillatory Integrals, Princeton University Press, Princeton, NJ, 1993. 